\title{
Inequalities between Power Means and Convex Combinations of the Harmonic and Logarithmic Means
}

\author{
Wei-Mao Qian' ${ }^{1}$ and Zhong-Hua Shen ${ }^{2}$ \\ ${ }^{1}$ School of Distance Education, Huzhou Broadcast and TV University, Huzhou 313000, China \\ ${ }^{2}$ Department of Mathematics, Hangzhou Normal University, Hangzhou 310036, China
}

Correspondence should be addressed to Wei-Mao Qian, qwm661977@126.com

Received 10 October 2011; Accepted 1 December 2011

Academic Editor: Md. Sazzad Chowdhury

Copyright (c) 2012 W.-M. Qian and Z.-H. Shen. This is an open access article distributed under the Creative Commons Attribution License, which permits unrestricted use, distribution, and reproduction in any medium, provided the original work is properly cited.

We prove that $\alpha H(a, b)+(1-\alpha) L(a, b)>M_{(1-4 \alpha) / 3}(a, b)$ for $\alpha \in(0,1)$ and all $a, b>0$ with $a \neq b$ if and only if $\alpha \in[1 / 4,1)$ and $\alpha H(a, b)+(1-\alpha) L(a, b)<M_{(1-4 \alpha) / 3}(a, b)$ if and only if $\alpha \in(0,3 \sqrt{345} / 80-11 / 16)$, and the parameter $(1-4 \alpha) / 3$ is the best possible in either case. Here, $H(a, b)=2 a b /(a+b), L(a, b)=(a-b) /(\log a-\log b)$, and $M_{p}(a, b)=\left(\left(a^{p}+b^{p}\right) / 2\right)^{1 / p}(p \neq 0)$ and $M_{0}(a, b)=\sqrt{a b}$ are the harmonic, logarithmic, and $p$ th power means of $a$ and $b$, respectively.

\section{Introduction}

The classical logarithmic mean $L(a, b)$ of two positive real numbers $a$ and $b$ with $a \neq b$ is defined by

$$
L(a, b)=\frac{a-b}{\log a-\log b}
$$

In the recent past, the bivariate means have been the subject of intensive research. In particular, many remarkable inequalities for $L(a, b)$ can be found in the literature [1-21]. It might be surprising that the logarithmic mean has applications in physics, economics, and even in meteorology [22-24]. In [22] the authors study a variant of Jensen's functional equation involving the logarithmic mean, which appears in a heat conduction problem. A representation of $L(a, b)$ as an infinite product and an iterative algorithm for computing it as the common limit of two sequences of special geometric and arithmetic means are given in [4]. 
In $[25,26]$ it is shown that $L(a, b)$ can be expressed in terms of Gauss hypergeometric function ${ }_{2} F_{1}$. And, in [26] the authors prove that the reciprocal of the logarithmic mean is strictly totally positive; that is, every $n \times n$ determinant with elements $1 / L\left(a_{i}, b_{i}\right)$, where $0<a_{1}<a_{2}<\cdots<a_{n}$ and $0<b_{1}<b_{2}<\cdots<b_{n}$, is positive for all $n \geq 1$.

Let $G(a, b)=\sqrt{a b}, H(a, b)=2 a b /(a+b), I(a, b)=1 / e\left(a^{a} / b^{b}\right)^{1 /(a-b)}, A(a, b)=(a+$ $b) / 2, M_{p}(a, b)=\left(\left(a^{p}+b^{p}\right) / 2\right)^{1 / p}(p \neq 0)$ and $M_{0}(a, b)=\sqrt{a b}$, and $L_{p}(a, b)=\left(a^{p+1}+b^{p+1}\right) /\left(a^{p}+\right.$ $\left.b^{p}\right)$ be the geometric, harmonic, identric, arithmetic, $p$ th power, and $p$ th Lehmer means of two positive numbers $a$ and $b$, respectively. Then it is well known that both $M_{p}(a, b)$ and $L_{p}(a, b)$ are continuous and strictly increasing with respect to $p \in \mathbb{R}$ for fixed $a, b>0$ with $a \neq b$, and the inequalities

$$
\begin{aligned}
\min \{a, b\} & <H(a, b)=M_{-1}(a, b)=L_{-1}(a, b)<G(a, b)=M_{0}(a, b)=L_{-1 / 2}(a, b) \\
& <L(a, b)<I(a, b)<A(a, b)=M_{1}(a, b)=L_{0}(a, b)<\max \{a, b\}
\end{aligned}
$$

hold for all $a, b>0$ with $a \neq b$.

In [4], Carlson proves that the double inequality

$$
\sqrt{\frac{G(a, b)(A(a, b)+G(a, b))}{2}}<L(a, b)<\frac{1}{2}(A(a, b)+G(a, b))
$$

holds for all $a, b>0$ with $a \neq b$.

In [5], Lin finds the best possible upper and lower power bounds for the logarithmic mean as follows:

$$
M_{0}(a, b)<L(a, b)<M_{1 / 3}(a, b)
$$

for all $a, b>0$ with $a \neq b$.

In [9], Sándor establishes that

$$
\sqrt{G(a, b) I(a, b)}<L(a, b)<A(a, b)+G(a, b)-I(a, b)
$$

for all $a, b>0$ with $a \neq b$. follows:

In [27], Alzer gives the optimal Lehmer mean bounds for $L,(L I)^{1 / 2}$, and $(L+I) / 2$ as

$$
\begin{gathered}
L_{-1 / 3}(a, b)<L(a, b)<L_{0}(a, b) \\
L_{-1 / 4}(a, b)<\sqrt{L(a, b) I(a, b)}<L_{0}(a, b) \\
L_{-1 / 4}(a, b)<\frac{1}{2}(L(a, b)+I(a, b))<L_{0}(a, b)
\end{gathered}
$$

for all $a, b>0$ with $a \neq b$. 
The following sharp bounds for $(L I)^{1 / 2}$ and $(L+I) / 2$ in terms of power mean are presented in [28]:

$$
\begin{gathered}
M_{0}(a, b)<\sqrt{L(a, b) I(a, b)}<M_{1 / 2}(a, b), \\
M_{\log 2 /(1+\log 2)}(a, b)<\frac{1}{2}(L(a, b)+I(a, b))<M_{1 / 2}(a, b)
\end{gathered}
$$

for all $a, b>0$ with $a \neq b$.

In $[29,30]$, the authors obtain the sharp bounds for the products $A^{\alpha}(a, b) L^{1-\alpha}(a, b)$ and $G^{\alpha}(a, b) L^{1-\alpha}(a, b)$ and the sum $\alpha A(a, b)+(1-\alpha) L(a, b)$ in terms of power mean as follows:

$$
\begin{gathered}
M_{0}(a, b)<A^{\alpha}(a, b) L^{1-\alpha}(a, b)<M_{(1+2 \alpha) / 3}(a, b), \\
M_{0}(a, b)<G^{\alpha}(a, b) L^{1-\alpha}(a, b)<M_{(1-\alpha) / 3}(a, b), \\
M_{\log 2 /(\log 2-\log \alpha)}(a, b)<\alpha A(a, b)+(1-\alpha) L(a, b)<M_{(1+2 \alpha) / 3}(a, b)
\end{gathered}
$$

for any $\alpha \in(0,1)$ and all $a, b>0$ with $a \neq b$.

In [31], Zhu presents some bounds for $I(a, b)$ in terms of $A(a, b)$ and $L(a, b)$ and $L(a, b)$ in terms of $G(a, b)$ and $I(a, b)$.

In [32], Chu et al. prove that the double inequality $\alpha A(a, b)+(1-\alpha) H(a, b)<P(a, b)<$ $\beta A(a, b)+(1-\beta) H(a, b)$ holds for all $a, b>0$ with $a \neq b$ if and only if $\alpha \leq 2 / \pi$ and $\beta \geq 5 / 6$.

It is the aim of this paper to give the optimal power mean bounds for the convex combination of harmonic and logarithmic means. Our main result is the following theorem.

Theorem 1.1. For $\alpha \in(0,1)$ and all $a, b>0$ with $a \neq b$, one has

(1) $\alpha H(a, b)+(1-\alpha) L(a, b)>M_{(1-4 \alpha) / 3}(a, b)$ if and only if $\alpha \in[1 / 4,1)$;

(2) $\alpha H(a, b)+(1-\alpha) L(a, b)<M_{(1-4 \alpha) / 3}(a, b)$ if and only if $\alpha \in(0,3 \sqrt{345} / 80-11 / 16)$.

In particular, the parameter $(1-4 \alpha) / 3$ is the best possible in either case.

\section{Lemmas}

In order to establish our main result we need to establish four lemmas, which we present in this section.

Lemma 2.1. Let $\alpha \in(1 / 4,1), p=(1-4 \alpha) / 3 \in(-1,0)$, and $g(t)=-4 \alpha p(p+1)^{2}(p+2) t^{p-1}+2(1-$ $\alpha) p^{2}\left(1-p^{2}\right) t^{p-2}+2(1-\alpha) p(1-p)^{2}(2-p) t^{p-3}+12(1-\alpha)(1-p)$. Then $g(t)>0$ for $t \in[1,+\infty)$.

Proof. Simple computations lead to

$$
\begin{aligned}
g(1)= & \frac{64}{81}(1-\alpha)^{2}\left(56 \alpha^{2}+23 \alpha+11\right)>0, \\
\lim _{t \rightarrow+\infty} g(t)= & 12(1-\alpha)(1-p)=8(1-\alpha)(1+2 \alpha)>0, \\
& g^{\prime}(t)=-2 p(1-p) t^{p-4} g_{1}(t),
\end{aligned}
$$


where

$$
\begin{gathered}
g_{1}(t)=-2 \alpha(p+1)^{2}(p+2) t^{2}+(1-\alpha) p(p+1)(2-p) t+(1-\alpha)(1-p)(2-p)(3-p), \\
g_{1}(1)=\frac{4}{27}(1-\alpha)\left(148 \alpha^{2}-11 \alpha+25\right)>0, \\
\lim _{t \rightarrow+\infty} g_{1}(t)=-\infty \\
g_{1}^{\prime}(t)=-4 \alpha(p+1)^{2}(p+2) t+(1-\alpha) p(p+1)(2-p) \\
=-\frac{4}{27}(1-\alpha)^{2}[16 \alpha(7-4 \alpha) t+(4 \alpha-1)(4 \alpha+5)]<0
\end{gathered}
$$

for $t \in[1,+\infty)$.

Inequality (2.6) implies that $g_{1}(t)$ is strictly decreasing in $[1,+\infty)$. Then (2.4) and (2.5) lead to the conclusion that there exists $\lambda_{1}>1$ such that $g_{1}(t)>0$ for $t \in\left[1, \lambda_{1}\right)$ and $g_{1}(t)<0$ for $t \in\left(\lambda_{1},+\infty\right)$. It follows from (2.3) that $g(t)$ is strictly increasing in $\left[1, \lambda_{1}\right]$ and strictly decreasing in $\left[\lambda_{1},+\infty\right)$.

Therefore, Lemma 2.1 follows from (2.1) and (2.2) together with the piecewise monotonicity of $g(t)$.

Lemma 2.2. Let $\alpha \in(1 / 4,1), p=(1-4 \alpha) / 3 \in(-1,0)$, and $h(t)=-(1-\alpha)(p+1)(p+2)^{2}(p+$ 3) $t^{p}+(p+1)\left(p^{3}-\alpha p^{3}-19 \alpha p^{2}+3 p^{2}-34 \alpha p+2 p-8 \alpha\right) t^{p-1}+(1-\alpha) p\left(p^{3}-8 p^{2}-p+4\right) t^{p-2}+(1-$ $\alpha)(1-p)\left(p^{3}+5 p^{2}-14 p+4\right) t^{p-3}+4(1-\alpha)(7-4 p)-4 p(1-\alpha) t^{-1}+4 \alpha(1+p) t^{-2}$. Then $h(t)>0$ for $t \in[1,+\infty)$.

Proof. Let

$$
h_{1}(t)=t^{3-p} h(t)
$$

Then simple computations lead to

$$
\begin{aligned}
h_{1}(1)= & \frac{16}{27}(1-\alpha)\left(80 \alpha^{2}+110 \alpha-1\right)>0, \\
h_{1}^{\prime}(t)= & -3(1-\alpha)(p+1)(p+2)^{2}(p+3) t^{2}+2(p+1) \\
& \times\left(p^{3}-\alpha p^{3}-19 \alpha p^{2}+3 p^{2}-34 \alpha p+2 p-8 \alpha\right) t+(1-\alpha) p\left(p^{3}-8 p^{2}-p+4\right) \\
& +4(1-\alpha)(7-4 p)(3-p) t^{2-p}-4 p(1-\alpha)(2-p) t^{1-p}+4 \alpha\left(1-p^{2}\right) t^{-p}, \\
h_{1}^{\prime}(1)= & \frac{32}{27}(1-\alpha)\left(-16 \alpha^{3}+38 \alpha^{2}+176 \alpha-9\right)>0,
\end{aligned}
$$


Journal of Applied Mathematics

$$
\begin{aligned}
h_{1}^{\prime \prime}(t)= & -6(1-\alpha)(p+1)(p+2)^{2}(p+3) t+2(p+1) \\
& \times\left(p^{3}-\alpha p^{3}-19 \alpha p^{2}+3 p^{2}-34 \alpha p+2 p-8 \alpha\right) \\
& +4(1-\alpha)(7-4 p)(3-p)(2-p) t^{1-p} \\
& -4 p(1-\alpha)(2-p)(1-p) t^{-p}-4 \alpha p\left(1-p^{2}\right) t^{-p-1}, \\
h_{1}^{\prime \prime}(1)= & \frac{8}{81}(1-\alpha)\left(-128 \alpha^{4}+896 \alpha^{3}+288 \alpha^{2}+5294 \alpha-437\right)>0, \\
h_{1}^{\prime \prime \prime}(t)= & -6(1-\alpha)(p+1)(p+2)^{2}(p+3)+4(1-\alpha)(7-4 p)(3-p)(2-p)(1-p) t^{-p} \\
& +4 p^{2}(1-\alpha)(2-p)(1-p) t^{-p-1}+4 \alpha p(1+p)^{2}(1-p) t^{-p-2}, \\
h_{1}^{\prime \prime \prime}(1)= & \frac{8}{81}(1-\alpha)\left(576 \alpha^{4}+3872 \alpha^{3}+660 \alpha^{2}+6612 \alpha-785\right)>0, \\
h_{1}^{(4)}(t)= & -4 p(1-p) t^{-p-3} h_{2}(t),
\end{aligned}
$$

where

$$
\begin{aligned}
h_{2}(t) & =(1-\alpha)(7-4 p)(3-p)(2-p) t^{2}+(1-\alpha) p(2-p)(p+1) t+\alpha(p+1)^{2}(p+2), \\
h_{2}(1) & =\frac{4}{27}(1-\alpha)\left(96 \alpha^{3}+232 \alpha^{2}+388 \alpha+175\right)>0, \\
h_{2}^{\prime}(t) & =2(1-\alpha)(7-4 p)(3-p)(2-p) t+(1-\alpha) p(2-p)(p+1) \\
& \geq h_{2}^{\prime}(1)=\frac{4}{9}(1-\alpha)(5+4 \alpha)\left(12 \alpha^{2}+31 \alpha+23\right)>0
\end{aligned}
$$

for $t \in[1,+\infty)$.

From inequalities (2.13) we clearly see that $h_{2}(t)>0$ for $t \in[1,+\infty)$. Then (2.12) leads to the conclusion that $h_{1}^{\prime \prime \prime}(t)$ is strictly increasing in $[1,+\infty)$.

Therefore, Lemma 2.2 follows from (2.7)-(2.11) and the monotonicity of $h_{1}^{\prime \prime \prime}(t)$.

Lemma 2.3. Let $\alpha \in(0,1), p=(1-4 \alpha) / 3, \lambda_{0}=3 \sqrt{345} / 80-11 / 16=0.00903 \ldots$, and $f(t)=$ $2 \alpha\left(1-t^{p+1}\right) t \log ^{2} t+(1-\alpha)\left(1+t^{p-1}\right)(1+t)^{2} t \log t+(1-\alpha)(1+t)^{2}(1-t)\left(t^{p}+1\right)$. Then the following two statements are true:

(1) if $\alpha \in(1 / 4,1)$, then $f(t)>0$ for $t \in(1,+\infty)$;

(2) if $\alpha \in\left(0, \lambda_{0}\right]$, then $f(t)<0$ for $t \in(1,+\infty)$. 
Proof. Let $f_{1}(t)=t^{-p} f^{\prime \prime}(t), f_{2}(t)=t^{p+2} f_{1}^{\prime}(t), f_{3}(t)=t^{4-p} f_{2}^{\prime \prime \prime}(t), f_{4}(t)=t^{p+2} f_{3}^{\prime \prime \prime}(t)$ and $f_{5}(t)=$ $t^{4-p} f_{4}^{\prime \prime \prime}(t)$. Then simple computations lead to

$$
\begin{aligned}
& f(1)=0, \\
& f^{\prime}(t)=2 \alpha\left[1-(p+2) t^{p+1}\right] \log ^{2} t \\
& +\left[(p+2-\alpha p-6 \alpha) t^{p+1}+2(1-\alpha)(p+1) t^{p}\right. \\
& \left.+(1-\alpha) p t^{p-1}+3(1-\alpha) t^{2}+4(1-\alpha) t+3 \alpha+1\right] \log t \\
& -(1-\alpha)\left[(p+3) t^{p+2}+(p+1) t^{p+1}-(p+3) t^{p}-(p+1) t^{p-1}+2 t^{2}-2\right], \\
& f^{\prime}(1)=0 \text {, } \\
& f_{1}(t)=-2 \alpha(p+1)(p+2) \log ^{2} t \\
& +\left[\left(p^{2}-\alpha p^{2}+3 p-11 \alpha p-14 \alpha+2\right)+2(1-\alpha) p(p+1) t^{-1}\right. \\
& \left.-(1-\alpha) p(1-p) t^{-2}+6(1-\alpha) t^{1-p}+4(1-\alpha) t^{-p}+4 \alpha p t^{-1-p}\right] \log t \\
& -(1-\alpha)(p+2)(p+3) t+(1-\alpha)\left(p^{2}+5 p+2\right) t^{-1} \\
& +(1-\alpha)\left(p^{2}+p-1\right) t^{-2}-(1-\alpha) t^{1-p}+4(1-\alpha) t^{-p}+(1+3 \alpha) t^{-1-p} \\
& -(1-\alpha) p^{2}-(1-\alpha) p-5 \alpha+1 \text {, } \\
& f_{1}(1)=0 \text {, } \\
& f_{2}(t)=-\left[4 \alpha(p+1)(p+2) t^{p+1}+2(1-\alpha) p(p+1) t^{p}-2(1-\alpha) p(1-p) t^{p-1}\right. \\
& \left.-6(1-\alpha)(1-p) t^{2}+4(1-\alpha) p t+4 \alpha(1+p)\right] \log t \\
& -(1-\alpha)(p+2)(p+3) t^{p+2}+\left(p^{2}-\alpha p^{2}+3 p-11 \alpha p-14 \alpha+2\right) t^{p+1} \\
& +(1-\alpha)\left(p^{2}-3 p-2\right) t^{p}-(1-\alpha)\left(p^{2}+3 p-2\right) t^{p-1}+(1-\alpha)(p+5) t^{2} \\
& +4(1-\alpha)(1-p) t+\alpha-3 \alpha p-p-1, \\
& f_{2}(1)=0 \text {, } \\
& f_{2}^{\prime}(t)=-\left[4 \alpha(p+1)^{2}(p+2) t^{p}+2(1-\alpha) p^{2}(p+1) t^{p-1}+2(1-\alpha) p(1-p)^{2} t^{p-2}\right. \\
& -12(1-\alpha)(1-p) t+4(1-\alpha) p] \log t-(1-\alpha)(p+2)^{2}(p+3) t^{p+1} \\
& +(p+1)\left(p^{2}-\alpha p^{2}-15 \alpha p+3 p-22 \alpha+2\right) t^{p}+(1-\alpha) p\left(p^{2}-5 p-4\right) t^{p-1} \\
& +(1-\alpha)(1-p)\left(p^{2}+5 p-2\right) t^{p-2}+4(1-\alpha)(4-p) t-4 \alpha(1+p) t^{-1} \\
& +4(1-\alpha)(1-2 p) \text {, } \\
& f_{2}^{\prime}(1)=0 \text {, }
\end{aligned}
$$


Journal of Applied Mathematics

$$
\begin{aligned}
f_{2}^{\prime \prime}(t)= & -\left[4 \alpha p(p+1)^{2}(p+2) t^{p-1}-2(1-\alpha) p^{2}\left(1-p^{2}\right) t^{p-2}-2(1-\alpha) p(1-p)^{2}\right. \\
& \left.\times(2-p) t^{p-3}-12(1-\alpha)(1-p)\right] \log t-(1-\alpha)(p+1)(p+2)^{2} \\
& \times(p+3) t^{p}+(p+1)\left(p^{3}-\alpha p^{3}-19 \alpha p^{2}+3 p^{2}-34 \alpha p+2 p-8 \alpha\right) t^{p-1} \\
& +(1-\alpha) p\left(p^{3}-8 p^{2}-p+4\right) t^{p-2}+(1-\alpha)(1-p)\left(p^{3}+5 p^{2}-14 p+4\right) t^{p-3} \\
& -4(1-\alpha) p t^{-1}+4 \alpha(1+p) t^{-2}+4(1-\alpha)(7-4 p) .
\end{aligned}
$$

(1) If $\alpha \in(1 / 4,1)$, then from (2.19) we note that

$$
f_{2}^{\prime \prime}(t)=g(t) \log t+h(t)
$$

where $g(t)$ and $h(t)$ are defined as in Lemmas 2.1 and 2.2, respectively.

Lemmas 2.1 and 2.2 together with (2.20) imply that $f_{2}^{\prime}(t)$ is strictly increasing in $[1,+\infty)$. Therefore, $f(t)>0$ for $t \in(1,+\infty)$ follows from $(2.14)-(2.18)$ and the monotonicity of $f_{2}^{\prime}(t)$.

(2) If $\alpha \in\left(0, \lambda_{0}\right]$, then from (2.19) we have

$$
\begin{aligned}
f_{2}^{\prime \prime}(1)= & \frac{16}{27}(1-\alpha)\left(80 \alpha^{2}+110 \alpha-1\right) \\
= & \frac{1280}{27}(1-\alpha)\left(\alpha-\lambda_{0}\right)\left(\alpha+\frac{3 \sqrt{345}}{80}+\frac{11}{16}\right) \leq 0, \\
f_{3}(t)= & {\left[4 \alpha p(1-p)(p+1)^{2}(p+2) t^{2}-2(1-\alpha) p^{2}\left(1-p^{2}\right)(2-p) t\right.} \\
& \left.-2(1-\alpha) p(1-p)^{2}(2-p)(3-p)\right] \log t-(1-\alpha) p(p+1)(p+2)^{2}(p+3) t^{3} \\
& +(p+1)\left(p^{4}-\alpha p^{4}-22 \alpha p^{3}+2 p^{3}-27 \alpha p^{2}-p^{2}-2 p+18 \alpha p+8 \alpha\right) t^{2} \\
& +(1-\alpha) p\left(p^{4}-12 p^{3}+15 p^{2}+8 p-8\right) t+(1-\alpha)(1-p)\left(p^{4}+4 p^{3}-35 p^{2}+50 p-12\right) \\
& +12(1-\alpha)(1-p) t^{3-p}+4 p(1-\alpha) t^{2-p}-8 \alpha(1+p) t^{1-p}, \\
f_{3}(1)= & \frac{32}{81}(1-\alpha)(1-4 \alpha)\left(80 \alpha^{2}+110 \alpha-1\right) \\
= & \frac{10240}{81}(1-\alpha)\left(\frac{1}{4}-\alpha\right)\left(\alpha-\lambda_{0}\right)\left(\alpha+\frac{3 \sqrt{345}}{80}+\frac{11}{16}\right) \leq 0,
\end{aligned}
$$




$$
\begin{aligned}
& f_{3}^{\prime}(t)=\left[8 \alpha p(1-p)(p+1)^{2}(p+2) t-2(1-\alpha) p^{2}\left(1-p^{2}\right)(2-p)\right] \log t \\
& -3(1-\alpha) p(p+1)(p+2)^{2}(p+3) t-2(p+1) \\
& \times\left(3 \alpha p^{4}-p^{4}+26 \alpha p^{3}-2 p^{3}+25 \alpha p^{2}+p^{2}-22 \alpha p+2 p-8 \alpha\right) t \\
& -(1-\alpha) p\left(p^{4}+8 p^{3}-17 p^{2}-4 p+8\right)-2(1-\alpha) p(1-p)^{2}(2-p)(3-p) t^{-1} \\
& +12(1-\alpha)(1-p)(3-p) t^{2-p}+4(1-\alpha) p(2-p) t^{1-p}-8 \alpha\left(1-p^{2}\right) t^{-p}, \\
& f_{3}^{\prime}(1)=\frac{8}{243}(1-\alpha)\left(3328 \alpha^{4}+128 \alpha^{3}-7248 \alpha^{2}+7118 \alpha-167\right) \\
& <\frac{8}{243}(1-\alpha)\left(3328 \lambda_{0}^{4}+128 \lambda_{0}^{3}+7118 \lambda_{0}-167\right) \\
& <\frac{8}{243}(1-\alpha)\left[3328 \times(0.01)^{4}+128 \times(0.01)^{3}+7118 \times 0.01-167\right]<0 ， \\
& f_{3}^{\prime \prime}(t)=8 \alpha p(1-p)(p+1)^{2}(p+2) \log t-6(1-\alpha) p(p+1)(p+2)^{2}(p+3) t \\
& -2(1-\alpha) p^{2}\left(1-p^{2}\right)(2-p) t^{-1}+2(1-\alpha) p(1-p)^{2}(2-p)(3-p) t^{-2} \\
& +12(1-\alpha)(1-p)(2-p)(3-p) t^{1-p}+4(1-\alpha) p(1-p)(2-p) t^{-p} \\
& +8 \alpha p\left(1-p^{2}\right) t^{-1-p}-2(p+1) \\
& \times\left(7 \alpha p^{4}-p^{4}+34 \alpha p^{3}-2 p^{3}+21 \alpha p^{2}+p^{2}-30 \alpha p+2 p-8 \alpha\right), \\
& f_{3}^{\prime \prime}(1)=\frac{8}{243}(1-\alpha)(7-4 \alpha)\left(256 \alpha^{4}-64 \alpha^{3}-1152 \alpha^{2}+2066 \alpha-53\right) \\
& <\frac{8}{243}(1-\alpha)(7-4 \alpha)\left(256 \lambda_{0}^{4}+2066 \lambda_{0}-53\right) \\
& <\frac{8}{243}(1-\alpha)[7-4 \alpha)\left(256 \times(0.01)^{4}+2066 \times 0.01-53\right]<0, \\
& f_{4}(t)=-6(1-\alpha) p(p+1)(p+2)^{2}(p+3) t^{p+2}+8 \alpha p(1-p)(p+1)^{2}(p+2) t^{p+1} \\
& +2(1-\alpha) p^{2}\left(1-p^{2}\right)(2-p) t^{p}-4(1-\alpha) p(1-p)^{2}(2-p)(3-p) t^{p-1} \\
& +12(1-\alpha)(1-p)^{2}(2-p)(3-p) t^{2}-4(1-\alpha) p^{2}(1-p)(2-p) t \\
& -8 \alpha p(1-p)(1+p)^{2} \text {, } \\
& f_{4}(1)=\frac{8}{243}(1-\alpha)\left(-1024 \alpha^{4}+21952 \alpha^{3}-10968 \alpha^{2}+13474 \alpha-835\right) \\
& <\frac{8}{243}(1-\alpha)\left(21952 \lambda_{0}^{3}+13474 \lambda_{0}-835\right) \\
& <\frac{8}{243}(1-\alpha)\left[21952 \times(0.01)^{3}+13474 \times 0.01-835\right]<0, \\
& f_{4}^{\prime}(t)=-6(1-\alpha) p(p+1)(p+2)^{3}(p+3) t^{p+1}+8 \alpha p(1-p)(p+1)^{3}(p+2) t^{p} \\
& +2(1-\alpha) p^{3}\left(1-p^{2}\right)(2-p) t^{p-1}+4(1-\alpha) p(1-p)^{3}(2-p)(3-p) t^{p-2} \\
& +24(1-\alpha)(1-p)^{2}(2-p)(3-p) t-4(1-\alpha) p^{2}(1-p)(2-p),
\end{aligned}
$$


Journal of Applied Mathematics

$$
\begin{aligned}
& f_{4}^{\prime}(1)=\frac{8}{729}(1-\alpha)(7-4 \alpha)\left(-1024 \alpha^{4}+21952 \alpha^{3}-10968 \alpha^{2}+13474 \alpha-835\right) \\
& <\frac{8}{729}(1-\alpha)(7-4 \alpha)\left(21952 \lambda_{0}^{3}+13474 \lambda_{0}-835\right) \\
& <\frac{8}{729}(1-\alpha)(7-4 \alpha)\left[21952 \times(0.01)^{3}+13474 \times 0.01-835\right]<0 \text {, } \\
& f_{4}^{\prime \prime}(t)=-6(1-\alpha) p(p+1)^{2}(p+2)^{3}(p+3) t^{p}+8 \alpha p^{2}(1-p)(p+1)^{3}(p+2) t^{p-1} \\
& -2(1-\alpha) p^{3}(1+p)(1-p)^{2}(2-p) t^{p-2}-4(1-\alpha) p(1-p)^{3}(2-p)^{2}(3-p) t^{p-3} \\
& +24(1-\alpha)(1-p)^{2}(2-p)(3-p) \\
& f_{4}^{\prime \prime}(1)=\frac{32}{2187}(1-\alpha)\left(-4096 \alpha^{6}+136320 \alpha^{5}-241440 \alpha^{4}+383672 \alpha^{3}\right. \\
& \left.-209850 \alpha^{2}+100113 \alpha-7255\right) \\
& <\frac{32}{2187}(1-\alpha)\left(136320 \lambda_{0}^{5}+383672 \lambda_{0}^{3}+100113 \lambda_{0}-7255\right) \\
& <\frac{32}{2187}(1-\alpha)\left[136320 \times(0.01)^{5}+383672 \times(0.01)^{3}+100113 \times 0.01-7255\right] \\
& <0 \text {, } \\
& f_{5}(t)=-6(1-\alpha) p^{2}(p+1)^{2}(p+2)^{3}(p+3) t^{3}-8 \alpha p^{2}(1-p)^{2}(p+1)^{3}(p+2) t^{2} \\
& +2(1-\alpha) p^{3}(1+p)(1-p)^{2}(2-p)^{2} t+4(1-\alpha) p(1-p)^{3}(2-p)^{2}(3-p)^{2}, \\
& f_{5}(1)=\frac{32}{6561}(1-\alpha)(1-4 \alpha)\left(-4096 \alpha^{6}+173568 \alpha^{5}-190368 \alpha^{4}+439136 \alpha^{3}\right. \\
& \left.-191370 \alpha^{2}+96723 \alpha-8665\right) \\
& <\frac{32}{6561}(1-\alpha)(1-4 \alpha)\left(173568 \lambda_{0}^{5}+439136 \lambda_{0}^{3}+96723 \lambda_{0}-8665\right) \\
& <\frac{32}{6561}(1-\alpha)(1-4 \alpha)\left[173568 \times(0.01)^{5}+439136 \times(0.01)^{3}+96723 \times 0.01-8665\right] \\
& <0 \text {, } \\
& f_{5}^{\prime}(t)=-18(1-\alpha) p^{2}(p+1)^{2}(p+2)^{3}(p+3) t^{2}-16 \alpha p^{2}(1-p)^{2}(p+1)^{3}(p+2) t \\
& +2(1-\alpha) p^{3}(1+p)(1-p)^{2}(2-p)^{2}, \\
& f_{5}^{\prime}(1)=\frac{32}{6561}(1-\alpha)^{2}(1-4 \alpha)^{2}\left(-17408 \alpha^{4}+69920 \alpha^{3}-119136 \alpha^{2}+95282 \alpha-30845\right) \\
& <\frac{32}{6561}(1-\alpha)(1-4 \alpha)^{2}\left(69920 \lambda_{0}^{3}+95282 \lambda_{0}-30845\right) \\
& <\frac{32}{6561}(1-\alpha)(1-4 \alpha)^{2}\left(69920 \times(0.01)^{3}+95282 \times 0.01-30845\right) \\
& <0 \text {, }
\end{aligned}
$$




$$
\begin{aligned}
f_{5}^{\prime \prime}(t) & =-36(1-\alpha) p^{2}(p+1)^{2}(p+2)^{3}(p+3) t-16 \alpha p^{2}(1-p)^{2}(p+1)^{3}(p+2), \\
f_{5}^{\prime \prime}(1) & =\frac{128}{6561}(1-\alpha)^{3}(1-4 \alpha)^{2}(7-4 \alpha)\left(160 \alpha^{3}-1856 \alpha^{2}+3370 \alpha-2205\right) \\
& <\frac{128}{6561}(1-\alpha)^{3}(1-4 \alpha)^{2}(7-4 \alpha)\left(160 \lambda_{0}^{3}+3370 \lambda_{0}-2205\right) \\
& <\frac{128}{6561}(1-\alpha)^{3}(1-4 \alpha)^{2}(7-4 \alpha)\left(160 \times(0.01)^{3}+3370 \times 0.01-2205\right) \\
& <0, \\
f_{5}^{\prime \prime \prime}(t) & =-36(1-\alpha) p^{2}(p+1)^{2}(p+2)^{3}(p+3) \\
& =-\frac{128}{729}(5-2 \alpha)(1-4 \alpha)^{2}(1-\alpha)^{3}(7-4 \alpha)^{3}<0 .
\end{aligned}
$$

Inequalities (2.30) imply that $f_{5}^{\prime}(t)$ is strictly decreasing in $[1,+\infty)$. Then $(2.29)$ leads to the conclusion that $f_{5}(t)$ is strictly decreasing in $[1,+\infty)$.

It follows from (2.28) and the monotonicity of $f_{5}(t)$ that $f_{4}^{\prime \prime}(t)$ is strictly decreasing in $[1,+\infty)$. Then inequalities (2.25)-(2.27) lead to the conclusion that $f_{4}(t)<0$ for $t \in[1,+\infty)$. Thus, $f_{3}^{\prime \prime}(t)$ is strictly decreasing in $[1,+\infty)$.

From inequalities (2.22)-(2.24) and the monotonicity of $f_{3}^{\prime \prime}(t)$ we clearly see that $f_{3}(t)<$ 0 for $t \in(1,+\infty)$. Thus, $f_{2}^{\prime \prime}(t)$ is strictly decreasing in $[1,+\infty)$.

It follows from (2.17) and (2.18) and inequality (2.21) together with the monotonicity of $f_{2}^{\prime \prime}(t)$ that $f_{2}(t)<0$ for $t \in(1,+\infty)$, which implies that $f_{1}(t)$ is strictly decreasing in $[1,+\infty)$. $f_{1}(t)$.

Therefore, $f(t)<0$ for $t \in(1,+\infty)$ follows from (2.14)-(2.16) and the monotonicity of

Lemma 2.4. $3 t^{4}-4 t\left(2 t^{2}-t+2\right) \log t-3>0$ for $t>1$.

Proof. Let

$$
J(t)=3 t^{4}-4 t\left(2 t^{2}-t+2\right) \log t-3 .
$$

Then simple computations lead to

$$
\begin{aligned}
& J(1)=0 \\
& J^{\prime}(t)=4\left(3 t^{3}-2 t^{2}+t-2\right)-8\left(3 t^{2}-t+1\right) \log t \\
& J^{\prime}(1)=0 \\
& J^{\prime \prime}(t)=\frac{4}{t} J_{1}(t)
\end{aligned}
$$

where $J_{1}(t)=9 t^{3}-10 t^{2}+3 t-2-2(6 t-1) t \log t$,

$$
\begin{aligned}
& J_{1}(1)=0, \\
& J_{1}^{\prime}(t)=27 t^{2}-32 t+5-2(12 t-1) \log t, \\
& J_{1}^{\prime}(1)=0 \\
& J_{1}^{\prime \prime}(t)=\frac{2}{t} J_{2}(t),
\end{aligned}
$$


where $J_{2}(t)=27 t^{2}-12 t \log t-28 t+1$,

$$
\begin{aligned}
& J_{2}(1)=0, \\
& J_{2}^{\prime}(t)=54 t-12 \log t-40>0
\end{aligned}
$$

for $t>1$.

Therefore, Lemma 2.4 follows from (2.31)-(2.36).

\section{Proof of Theorem 1.1}

Proof of Theorem 1.1. For all $a, b>0$ with $a \neq b$, we first prove that

$$
\alpha H(a, b)+(1-\alpha) L(a, b)>M_{(1-4 \alpha) / 3}(a, b)
$$

for $\alpha \in[1 / 4,1)$,

$$
\alpha H(a, b)+(1-\alpha) L(a, b)<M_{(1-4 \alpha) / 3}(a, b)
$$

for $\alpha \in(0,3 \sqrt{345} / 80-11 / 16)$.

Without loss of generality, we assume that $a>b, t=a / b>1$ and $p=(1-4 \alpha) / 3$. We divide the proof into two cases.

Case $1(\alpha=1 / 4)$. Let $x=\sqrt{t}=\sqrt{a / b}>1$. Then we clearly see that

$$
\begin{aligned}
\alpha H(a, b)+(1-\alpha) L(a, b)-M_{(1-4 \alpha) / 3}(a, b) & =\frac{1}{4}[H(a, b)+3 L(a, b)]-\sqrt{a b} \\
& =\frac{b\left[3 x^{4}-4 x\left(2 x^{2}-x+2\right) \log x-3\right]}{8\left(x^{2}+1\right) \log x} .
\end{aligned}
$$

Therefore, inequality (3.1) follows from (3.3) and Lemma 2.4.

Case $2(\alpha \in(0,3 \sqrt{345} / 80-11 / 16) \cup(1 / 4,1))$. Then we have

$$
\begin{aligned}
\alpha H(a, b)+(1-\alpha) L(a, b)-M_{(1-4 \alpha) / 3}(a, b) & =\alpha H(a, b)+(1-\alpha) L(a, b)-M_{p}(a, b) \\
& =b\left[\frac{2 \alpha t}{t+1}+\frac{(1-\alpha)(t-1)}{\log t}-\left(\frac{t^{p}+1}{2}\right)^{1 / p}\right] .
\end{aligned}
$$

Let

$$
F(t)=\log \left[\frac{2 \alpha t}{t+1}+\frac{(1-\alpha)(t-1)}{\log t}\right]-\frac{1}{p} \log \left(\frac{t^{p}+1}{2}\right) .
$$


Then simple computations lead to

$$
\begin{gathered}
\lim _{t \rightarrow 1} F(t)=0, \\
F^{\prime}(t)=\frac{f(t)}{t(t+1)\left(t^{p}+1\right)\left[2 \alpha t \log t+(1-\alpha)\left(t^{2}-1\right)\right] \log t^{\prime}}
\end{gathered}
$$

where $f(t)$ is defined as in Lemma 2.3.

If $\alpha \in(1 / 4,1)$, then inequality (3.1) follows from (3.4)-(3.6) and Lemma 2.3(1). If $\alpha \in$ $(0,3 \sqrt{345} / 80-11 / 16)$, then inequality (3.2) follows from (3.4)-(3.6) and Lemma 2.3(2).

Next, we prove that the parameter $(1-4 \alpha) / 3$ in inequalities (3.1) and (3.2) is the best possible.

For any $\alpha \in(0,3 \sqrt{345} / 80-11 / 16) \cup(1 / 4,1), p \neq 0$, and $x>0$, one has

$$
\alpha H(1,1+x)+(1-\alpha) L(1,1+x)-M_{p}(1,1+x)=\frac{Q(x)}{2^{1 / p}(1+x / 2) \log (1+x)}
$$

where $Q(x)=2^{1 / p} \alpha(1+x) \log (1+x)+2^{1 / p}(1-\alpha) x(1+x / 2)-(1+x / 2) \log (1+x)\left[1+(1+x)^{p}\right]^{1 / p}$. Letting $x \rightarrow 0$ and making use of Taylor expansion, we get

$$
Q(x)=\frac{2^{1 / p}}{8}\left(\frac{1-4 \alpha}{3}-p\right) x^{3}+o\left(x^{3}\right)
$$

If $\alpha \in[1 / 4,1)$ and $p>(1-4 \alpha) / 3$, then (3.7) and (3.8) imply that there exists $\delta_{1}=$ $\delta_{1}(\alpha, p)>0$ such that $\alpha H(1,1+x)+(1-\alpha) L(1,1+x)<M_{p}(1,1+x)$ for $x \in\left(0, \delta_{1}\right)$. If $\alpha \in(0,3 \sqrt{345} / 80-11 / 16)$ and $p<(1-4 \alpha) / 3$, then (3.7) and (3.8) imply that there exists $\delta_{2}=\delta_{2}(\alpha, p)>0$ such that $\alpha H(1,1+x)+(1-\alpha) L(1,1+x)>M_{p}(1,1+x)$ for $x \in\left(0, \delta_{2}\right)$.

Finally, we prove that there exist $a_{1}, b_{1}, a_{2}, b_{2}>0$ with $a_{1} \neq b_{1}$ and $a_{2} \neq b_{2}$ such that $\alpha H\left(a_{1}, b_{1}\right)+(1-\alpha) L\left(a_{1}, b_{1}\right)<M_{(1-4 \alpha) / 3}\left(a_{1}, b_{1}\right)$ and $\alpha H\left(a_{2}, b_{2}\right)+(1-\alpha) L\left(a_{2}, b_{2}\right)>$ $M_{(1-4 \alpha) / 3}\left(a_{2}, b_{2}\right)$ for any $3 \sqrt{345} / 80-11 / 16<\alpha<1 / 4$.

If $3 \sqrt{345} / 80-11 / 16<\alpha<1 / 4$, then from the expression of $f_{2}^{\prime \prime}(1)$ in (2.21) we clearly see that $f_{2}^{\prime \prime}(1)>0$, which leads to the conclusion that there exists $\lambda>1$ such that

$$
f_{2}^{\prime \prime}(t)>0
$$

for $t \in[1, \lambda)$.

From (2.14)-(2.18) and inequality (3.9) we know that

$$
f(t)>0
$$

for $t \in(1, \lambda)$. Equations (3.4)-(3.6) and inequality (3.10) lead to the conclusion that $\alpha H(a, b)+$ $(1-\alpha) L(a, b)>M_{(1-4 \alpha) / 3}(a, b)$ for all $a / b \in(1, \lambda)$. 
On the other hand, simple computations lead to

$$
\begin{aligned}
& \lim _{x \rightarrow+\infty} \frac{M_{(1-4 \alpha) / 3}(1, x)}{\alpha H(1, x)+(1-\alpha) L(1, x)} \\
&=2^{3 /(4 \alpha-1)} \lim _{x \rightarrow+\infty} \frac{\left(1+x^{(4 \alpha-1) / 3}\right)^{3 /(1-4 \alpha)}}{2 \alpha /(x+1)+(1-\alpha)(1-1 / x) / \log x}=+\infty .
\end{aligned}
$$

Equation (3.11) implies that there exists $X=X(\alpha)>1$ such that $\alpha H(a, b)+(1-$ $\alpha) L(a, b)<M_{(1-4 \alpha) / 3}(a, b)$ for all $a / b \in(X,+\infty)$.

\section{Acknowledgment}

This work was supported by the Natural Science Foundation of Zhejiang Broadcast and TV University (Grant no. XKT-09G21).

\section{References}

[1] Y.-M. Chu and W.-F. Xia, "Two optimal double inequalities between power mean and logarithmic mean," Computers \& Mathematics with Applications, vol. 60, no. 1, pp. 83-89, 2010.

[2] Y.-F. Qiu, M.-K. Wang, Y.-M. Chu, and G.-D. Wang, "Two sharp inequalities for Lehmer mean, identric mean and logarithmic mean," Journal of Mathematical Inequalities, vol. 5, no. 3, pp. 301-306, 2011.

[3] Y.-M. Chu and B.-Y. Long, "Best possible inequalities between generalized logarithmic mean and classical means," Abstract and Applied Analysis, vol. 2010, Article ID 303286, 13 pages, 2010.

[4] B. C. Carlson, "The logarithmic mean," The American Mathematical Monthly, vol. 79, pp. 615-618, 1972.

[5] T. P. Lin, "The power mean and the logarithmic mean," The American Mathematical Monthly, vol. 81, pp. 879-883, 1974.

[6] A. O. Pittenger, "Inequalities between arithmetic and logarithmic means," Univerzitet u Beogradu. Publikacije Elektrotehničkog Fakulteta. Serija Matematika i Fizika, no. 678-715, pp. 15-18, 1980.

[7] A. O. Pittenger, "The symmetric, logarithmic and power means," Univerzitet u Beogradu. Publikacije Elektrotehničkog Fakulteta. Serija Matematika i Fizika, no. 678-715, pp. 19-23, 1980.

[8] J. Sándor, "On the identric and logarithmic means," Aequationes Mathematicae, vol. 40, no. 2-3, pp. 261-270, 1990.

[9] J. Sándor, “A note on some inequalities for means," Archiv der Mathematik, vol. 56, no. 5, pp. 471-473, 1991.

[10] J. Sándor, “On certain identities for means," Universitatis Babeş-Bolyai. Studia. Mathematica, vol. 38, no. 4, pp. 7-14, 1993.

[11] J. Sándor, "On certain inequalities for means," Journal of Mathematical Analysis and Applications, vol. 189, no. 2, pp. 602-606, 1995.

[12] J. Sándor, "On refinements of certain inequalities for means," Archivum Mathematicum, vol. 31, no. 4, pp. 279-282, 1995.

[13] J. Sándor, "On certain inequalities for means. II," Journal of Mathematical Analysis and Applications, vol. 199, no. 2, pp. 629-635, 1996.

[14] J. Sándor, "On certain inequalities for means. III," Archiv der Mathematik, vol. 76, no. 1, pp. 34-40, 2001.

[15] J. Sándor and I. Raşa, "Inequalities for certain means in two arguments," Nieuw Archief voor Wiskunde, vol. 15, no. 1-2, pp. 51-55, 1997.

[16] J. Sándor and T. Trif, "Some new inequalities for means of two arguments," International Journal of Mathematics and Mathematical Sciences, vol. 25, no. 8, pp. 525-532, 2001.

[17] Y.-M. Chu and W.-F. Xia, "Inequalities for generalized logarithmic means," Journal of Inequalities and Applications, vol. 2009, Article ID 763252, 7 pages, 2009.

[18] B.-Y. Long and Y.-M. Chu, "Optimal inequalities for generalized logarithmic, arithmetic, and geometric means," Journal of Inequalities and Applications, vol. 2010, Article ID 806825, 10 pages, 2010. 
[19] K. B. Stolarsky, “Generalizations of the logarithmic mean," Mathematics Magazine, vol. 48, no. 2, pp. 87-92, 1975.

[20] K. B. Stolarsky, “The power and generalized logarithmic means," The American Mathematical Monthly, vol. 87, no. 7, pp. 545-548, 1980.

[21] M. K. Vamanamurthy and M. Vuorinen, "Inequalities for means," Journal of Mathematical Analysis and Applications, vol. 183, no. 1, pp. 155-166, 1994.

[22] P. Kahlig and J. Matkowski, "Functional equations involving the logarithmic mean," Zeitschrift für Angewandte Mathematik und Mechanik, vol. 76, no. 7, pp. 385-390, 1996.

[23] A. O. Pittenger, "The logarithmic mean in $n$ variables," The American Mathematical Monthly, vol. 92, no. 2, pp. 99-104, 1985.

[24] G. Pólya and G. Szegö, Isoperimetric Inequalities in Mathematical Physics, Princeton University Press, Princeton, NJ, USA, 1951.

[25] B. C. Carlson, "Algorithms involving arithmetic and geometric means," The American Mathematical Monthly, vol. 78, pp. 496-505, 1971.

[26] B. C. Carlson and J. L. Gustafson, "Total positivity of mean values and hypergeometric functions," SIAM Journal on Mathematical Analysis, vol. 14, no. 2, pp. 389-395, 1983.

[27] H. Alzer, "Bestmögliche Abschötzungen für spezielle Mittelwerte," Zbornik Radova Prirodno-Matematichkog Fakulteta. Serija za Matematiku, vol. 23, no. 1, pp. 331-346, 1993.

[28] H. Alzer and S.-L. Qiu, "Inequalities for means in two variables," Archiv der Mathematik, vol. 80, no. 2, pp. 201-215, 2003.

[29] M.-Y. Shi, Y.-M. Chu, and Y.-P. Jiang, "Optimal inequalities among various means of two arguments," Abstract and Applied Analysis, vol. 2009, Article ID 694394, 10 pages, 2009.

[30] W.-F. Xia, Y.-M. Chu, and G.-D. Wang, "The optimal upper and lower power mean bounds for a convex combination of the arithmetic and logarithmic means," Abstract and Applied Analysis, vol. 2010, Article ID 604804, 9 pages, 2010.

[31] L. Zhu, "New inequalities for means in two variables," Mathematical Inequalities $\mathcal{E}$ Applications, vol. 11, no. 2, pp. 229-235, 2008.

[32] Y.-M. Chu, Y.-F. Qiu, M.-K. Wang, and G.-D. Wang, "The optimal convex combination bounds of arithmetic and harmonic means for the Seiffert's mean," Journal of Inequalities and Applications, vol. 2010, Article ID 436457, 7 pages, 2010. 


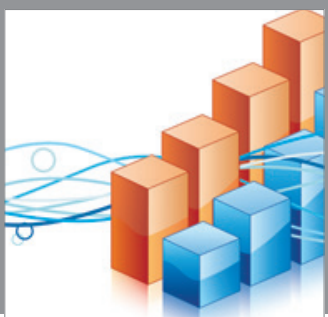

Advances in

Operations Research

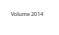

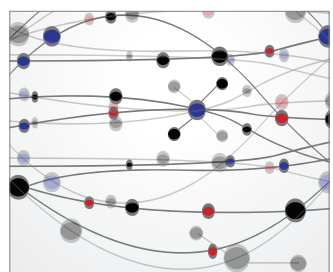

\section{The Scientific} World Journal
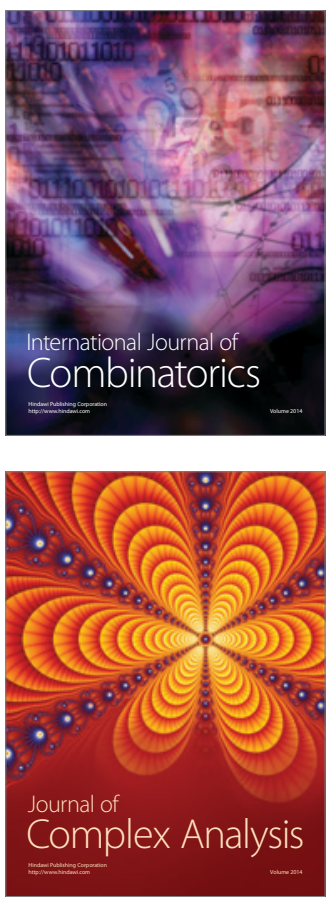

International Journal of

Mathematics and

Mathematical

Sciences
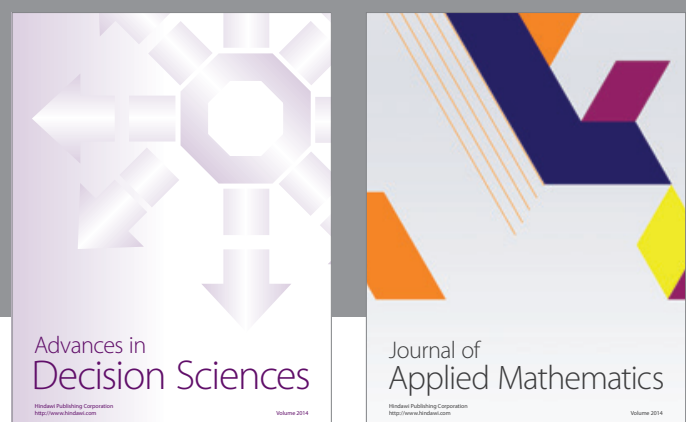

Journal of

Applied Mathematics
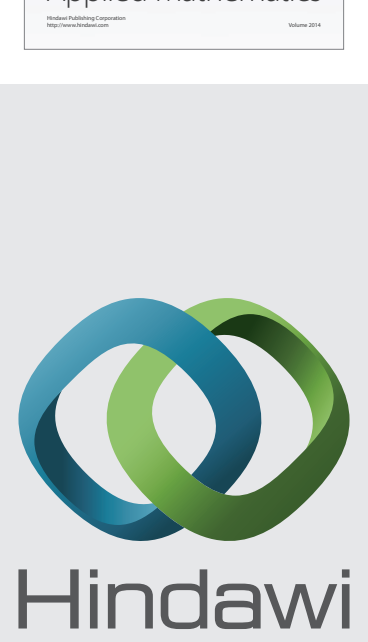

Submit your manuscripts at http://www.hindawi.com
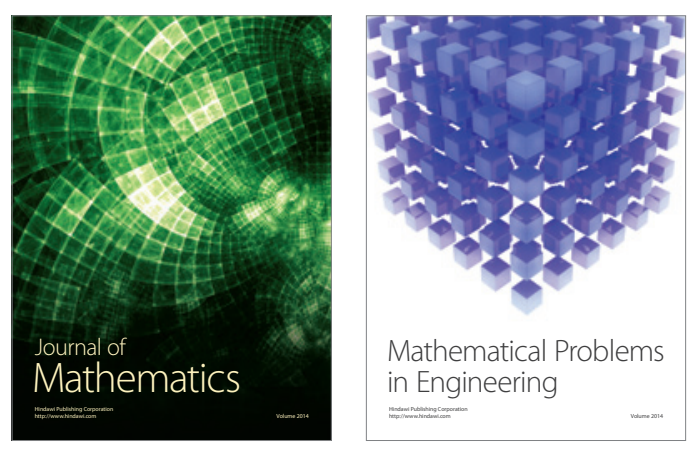

Mathematical Problems in Engineering
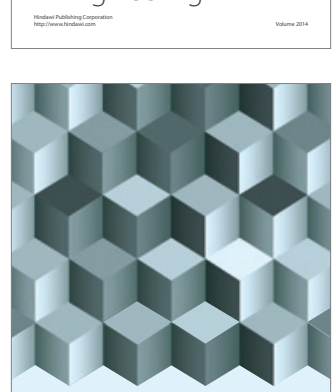

Journal of

Function Spaces
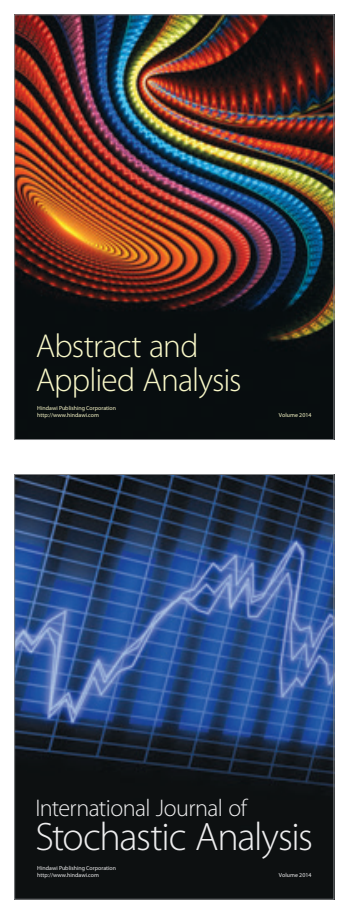

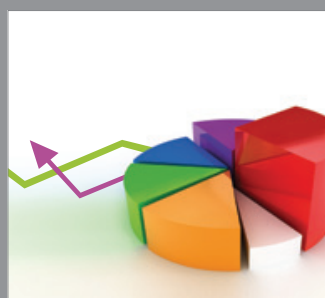

ournal of

Probability and Statistics

Promensencen
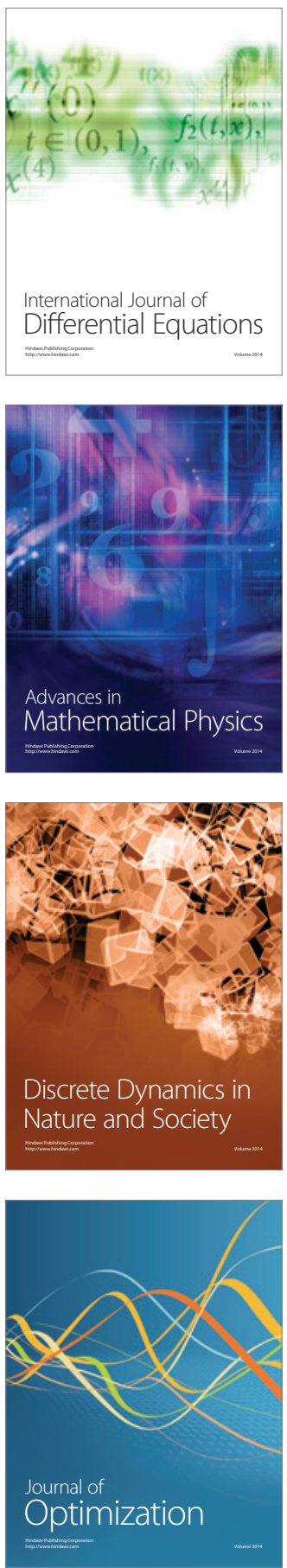